\title{
NMR Data
}

Synthesis and characterization of the magnetic zeolite Y-palladium-nickel ferrite by ultrasonic irradiation and investigation its catalytic activity in Suzuki-Miyaura cross coupling reaction

Modarres Dehghani, Azadeh Tadjarodi*, Sanaz Chamani

Research Laboratory of Inorganic Materials Synthesis ,Department of Chemistry, Iran University of Science and Technology, Narmak, Tehran, Iran Fax:+98 (21) 77491204; email: Tajarodi@iust.ac.ir

NMR spectra of the products are given in Table 4 in the main manuscript are presented here. 


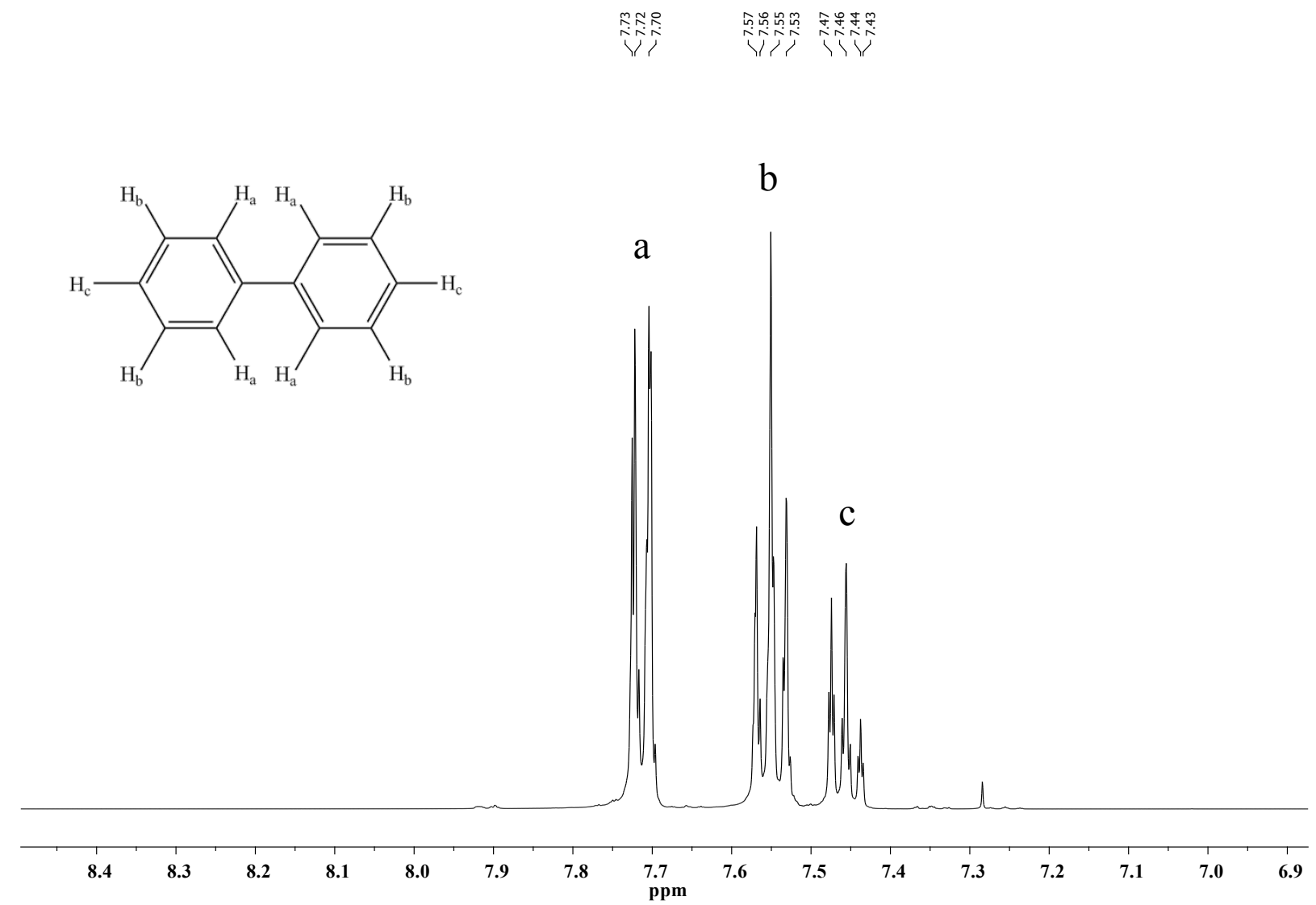

Figure S1. ${ }^{1} \mathrm{H}$ NMR spectrum of biphenyl (a) in $\mathrm{CDCl}_{3}$

Biphenyl: White solid, mp: $69-70{ }^{\circ} \mathrm{C}$ (lit. mp 69-71 $\left.{ }^{\circ} \mathrm{C}\right)$; FT-IR $\left(\mathrm{KBr} / \mathrm{cm}^{-1}\right)$ : 3020, 1565 , $1463,1412,1170,1010,726,697 ;{ }^{1} \mathrm{H}$ NMR (400 MHz, $\left.\mathrm{CDCl}_{3}, \mathrm{ppm}\right): \delta=7.46(\mathrm{~m}, 2 \mathrm{H})$, $7.55(\mathrm{~m}, 4 \mathrm{H}), 7.71(\mathrm{~m}, 4 \mathrm{H})$. 


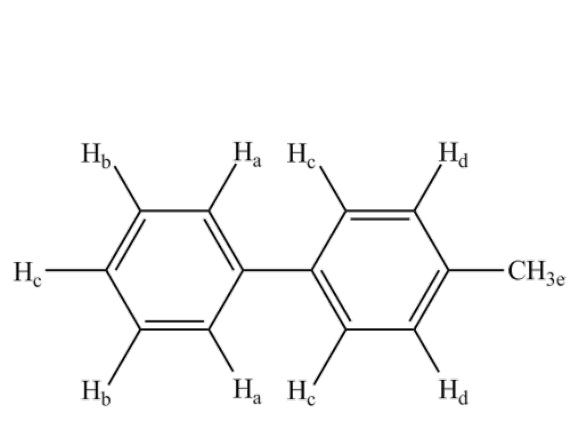

e
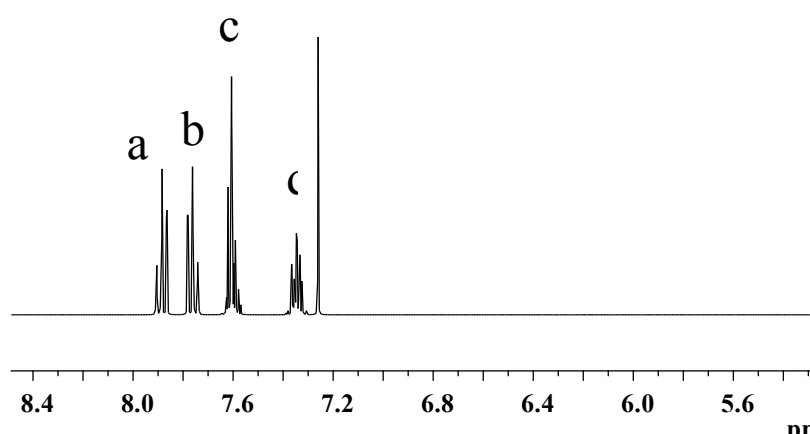

Figure S2. ${ }^{1} \mathrm{H}$ NMR spectrum of 4-methylbiphenyl (b) in $\mathrm{CDCl}_{3}$

4-Methylbiphenyl: White solid, mp: $43-44{ }^{\circ} \mathrm{C}$ (lit. mp 44-46 $\left.{ }^{\circ} \mathrm{C}\right)$; FT-IR $\left(\mathrm{KBr} / \mathrm{cm}^{-1}\right)$ : 3080, 3010, 2935, 2842, 1600, 1445, 1341, 1131, 1023, 706; ${ }^{1} \mathrm{H}$ NMR (400 MHz, $\left.\mathrm{CDCl}_{3}, \mathrm{ppm}\right)$ : $\delta=2.45\left(\mathrm{~s}, 3 \mathrm{H}, \mathrm{CH}_{3}\right), \delta=7.33(\mathrm{~m}, 2 \mathrm{H}), 7.37(\mathrm{~m} \mathrm{1H}), 7.59(\mathrm{~m}, 2 \mathrm{H}), 7.76(\mathrm{~m}, 2 \mathrm{H}), 7.88$ $(2 \mathrm{H})$. 


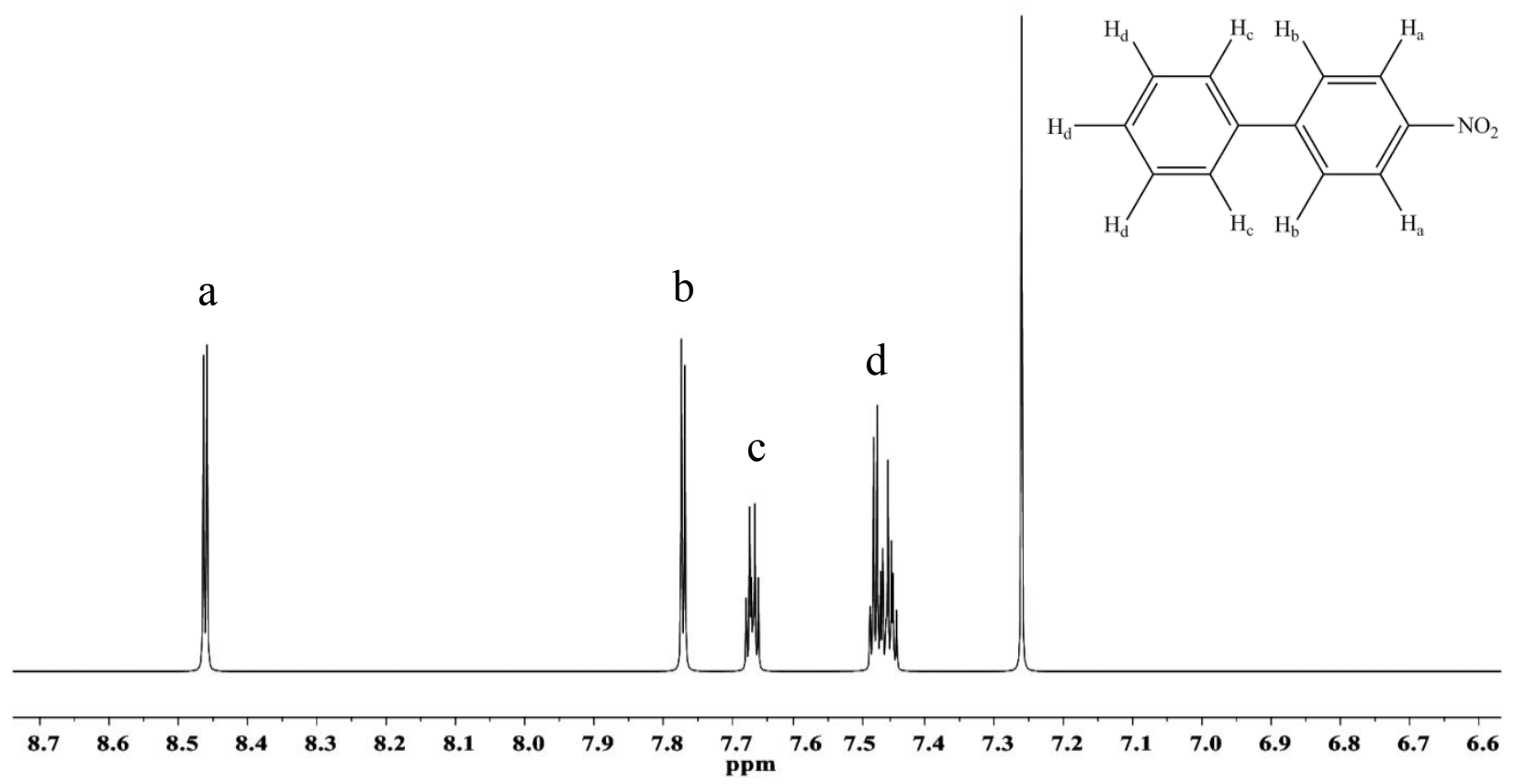

Figure S3. ${ }^{1} \mathrm{H}$ NMR spectrum of 4-nitrobiphenyl (c) in $\mathrm{CDCl}_{3}$

4-Nitrobiphenyl: Yellow solid, mp: $112-114{ }^{\circ} \mathrm{C}$ (lit.mp $\left.113-115{ }^{\circ} \mathrm{C}\right)$; FT-IR $\left(\mathrm{KBr} / \mathrm{cm}^{-1}\right)$ : $3112,3015,1613,1570,1445,1317,1083,1024,701 ;{ }^{1} \mathrm{H} \mathrm{NMR}\left(400 \mathrm{MHz}, \mathrm{CDCl}_{3}, \mathrm{ppm}\right)$ : $\delta=7.47(\mathrm{~m}, 3 \mathrm{H}), 7.67(\mathrm{~m} \mathrm{2H}), 7.77(\mathrm{~m}, 2 \mathrm{H}), 8.46(\mathrm{~m}, 2 \mathrm{H})$. 


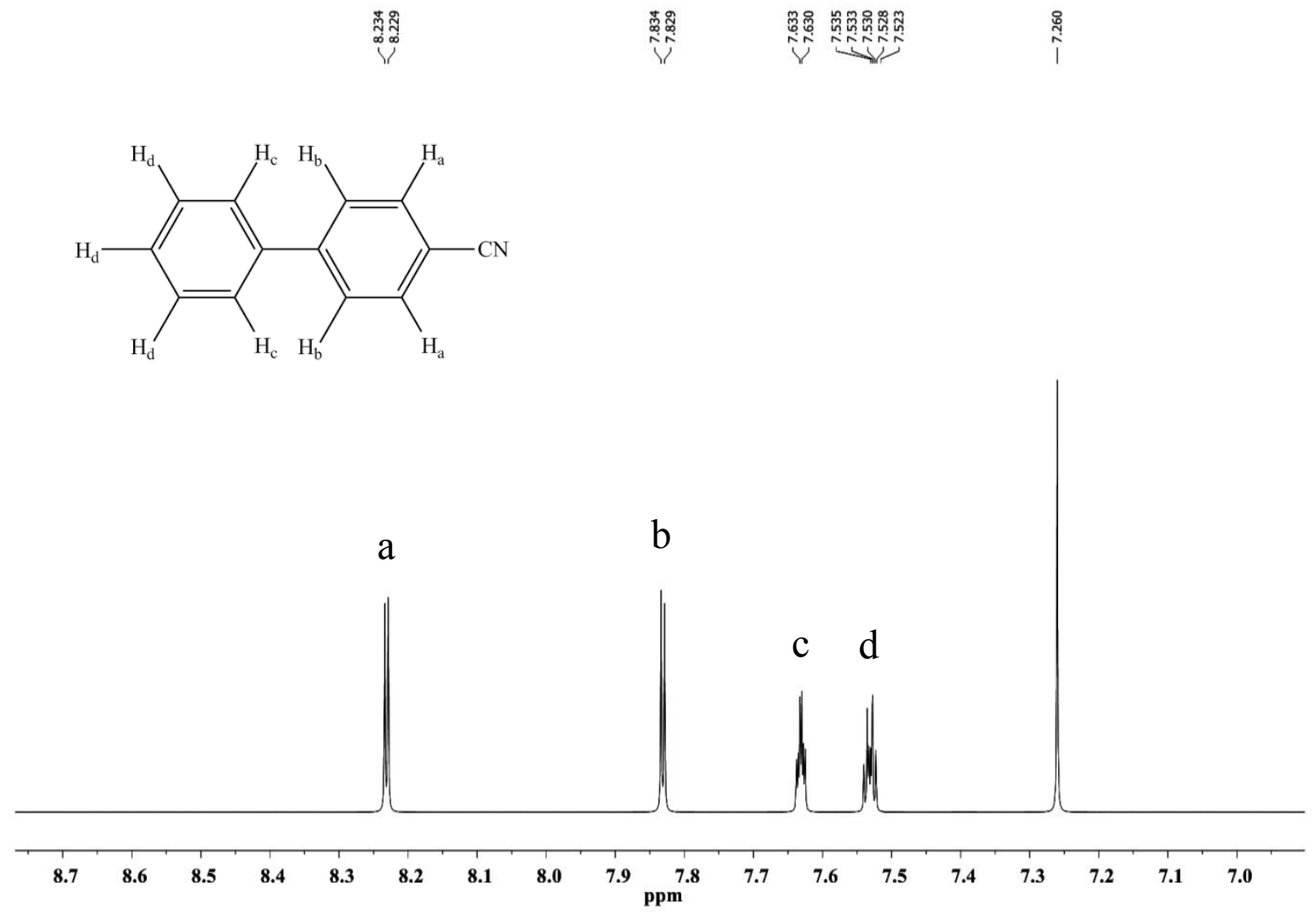

Figure S4. ${ }^{1} \mathrm{H}$ NMR spectrum of 4-phenylbenzonitrile (d) in $\mathrm{CDCl}_{3}$

4-Phenylbenzonitrile: White solid, mp: $84-86{ }^{\circ} \mathrm{C}$ (lit.mp $\left.84-88{ }^{\circ} \mathrm{C}\right)$; FT-IR $\left(\mathrm{KBr} / \mathrm{cm}^{-1}\right)$ : $3053,3015,2217,1642,1602,1445,1345,1091,1021,700 ;{ }^{1} \mathrm{H}$ NMR (400 MHz, $\mathrm{CDCl}_{3}$, ppm): $\delta=7.53(\mathrm{~m}, 3 \mathrm{H}), 7.63(\mathrm{~m} 2 \mathrm{H}), 7.83(\mathrm{~m}, 2 \mathrm{H}), 8.23(\mathrm{~m}, 2 \mathrm{H})$. 


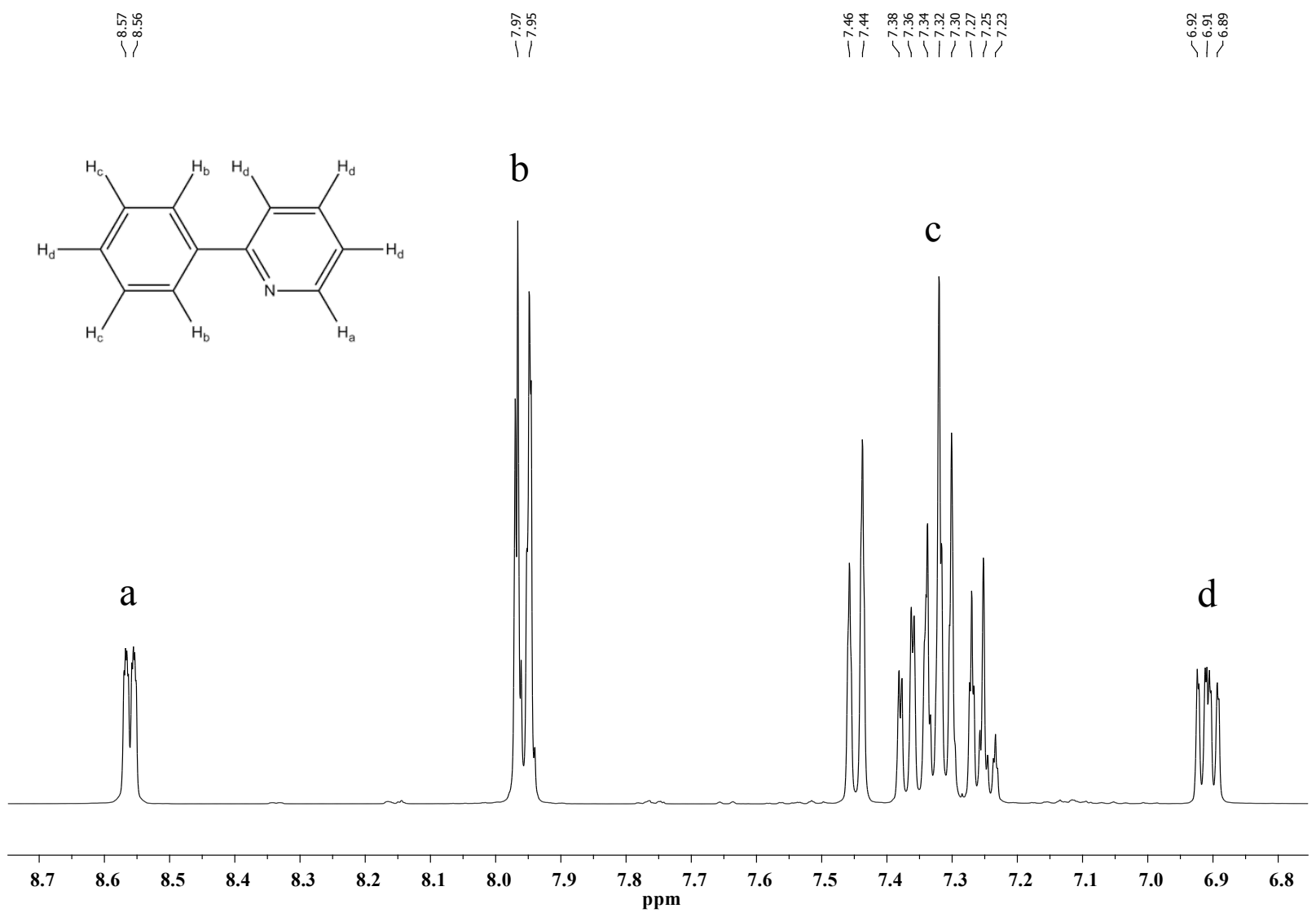

Figure S5. ${ }^{1} \mathrm{H}$ NMR spectrum of 2-phenylpyridine (e) in $\mathrm{CDCl}_{3}$

2-Phenylpyridine: Colorless oil, FT-IR (KBr/ $\left.\mathrm{cm}^{-1}\right): 3100,3056,3030,1741,1584,1570$, $1451,1431,1265,1021,812,727,700 ;{ }^{1} \mathrm{H} \mathrm{NMR}\left(400 \mathrm{MHz}, \mathrm{CDCl}_{3}, \mathrm{ppm}\right): \delta=6.89(\mathrm{~m}$, 1H), $7.28(\mathrm{~m}, 1 \mathrm{H}), 7.32(\mathrm{~m}, 2 \mathrm{H}), 7.37(\mathrm{~m}, 1 \mathrm{H}), 7.45(\mathrm{~m}, 1 \mathrm{H}), 7.96(\mathrm{~m}, 2 \mathrm{H}), 8.56(\mathrm{~m}, 1 \mathrm{H})$. 